\title{
Habitable Planets: Interior Dynamics and Long-Term Evolution
}

\author{
Paul J. Tackley ${ }^{1}$, Michael M. Ammann ${ }^{2}$, John P. Brodholt ${ }^{2}$, \\ David P. Dobson ${ }^{2}$ and Diana Valencia ${ }^{3}$ \\ ${ }^{1}$ Institute of Geophysics, Department of Earth Sciences, ETH Zurich, \\ Sonneggstrasse 4, 8092 Zurich, Switzerland \\ email: ptackley@ethz.ch \\ ${ }^{2}$ Dept. Earth Sciences, University College London, \\ Gower Street, London WC1E 6BT, UK \\ ${ }^{3}$ Dept. Earth, Atmospheric and Planetary Sciences, Massachusetts Institute of Technology, \\ Cambridge, MA 02139, USA
}

\begin{abstract}
Here, the state of our knowledge regarding the interior dynamics and evolution of habitable terrestrial planets including Earth and super-Earths is reviewed, and illustrated using state-of-the-art numerical models. Convection of the rocky mantle is the key process that drives the evolution of the interior: it causes plate tectonics, controls heat loss from the metallic core (which generates the magnetic field) and drives long-term volatile cycling between the atmosphere/ocean and interior. Geoscientists have been studying the dynamics and evolution of Earth's interior since the discovery of plate tectonics in the late 1960s and on many topics our understanding is very good, yet many first-order questions remain. It is commonly thought that plate tectonics is necessary for planetary habitability because of its role in long-term volatile cycles that regulate the surface environment. Plate tectonics is the surface manifestation of convection in the $2900-\mathrm{km}$ deep rocky mantle, yet exactly how plate tectonics arises is still quite uncertain; other terrestrial planets like Venus and Mars instead have a stagnant lithosphereessentially a single plate covering the entire planet. Nevertheless, simple scalings as well as more complex models indicate that plate tectonics should be easier on larger planets (super-Earths), other things being equal. The dynamics of terrestrial planets, both their surface tectonics and deep mantle dynamics, change over billions of years as a planet cools. Partial melting is a key process influencing solid planet evolution. Due to the very high pressure inside super-Earths' mantles the viscosity would normally be expected to be very high, as is also indicated by our density function theory (DFT) calculations. Feedback between internal heating, temperature and viscosity leads to a superadiabatic temperature profile and self-regulation of the mantle viscosity such that sluggish convection still occurs.
\end{abstract}

Keywords. Earth, planets and satellites: general, convection, methods: numerical

\section{Introduction}

The atmosphere will be the first feature (other than mass and radius) of extra-solar terrestrial planets to be observed, but the atmosphere of Earth - the prototypical habitable planet - has changed greatly over billions of years due largely to coupling with the solid interior via long-term volatile cycles, the most important of which involve water and carbon. Thus, the dynamics of a habitable planets mantle and lithosphere, including its tectonic mode (plate tectonics or a stagnant lid) is important to understand. In this paper, the state of our knowledge regarding the interior dynamics and evolution of habitable terrestrial planets, including Earth and super-Earths, is reviewed, and illustrated by state-of-the-art numerical models. 


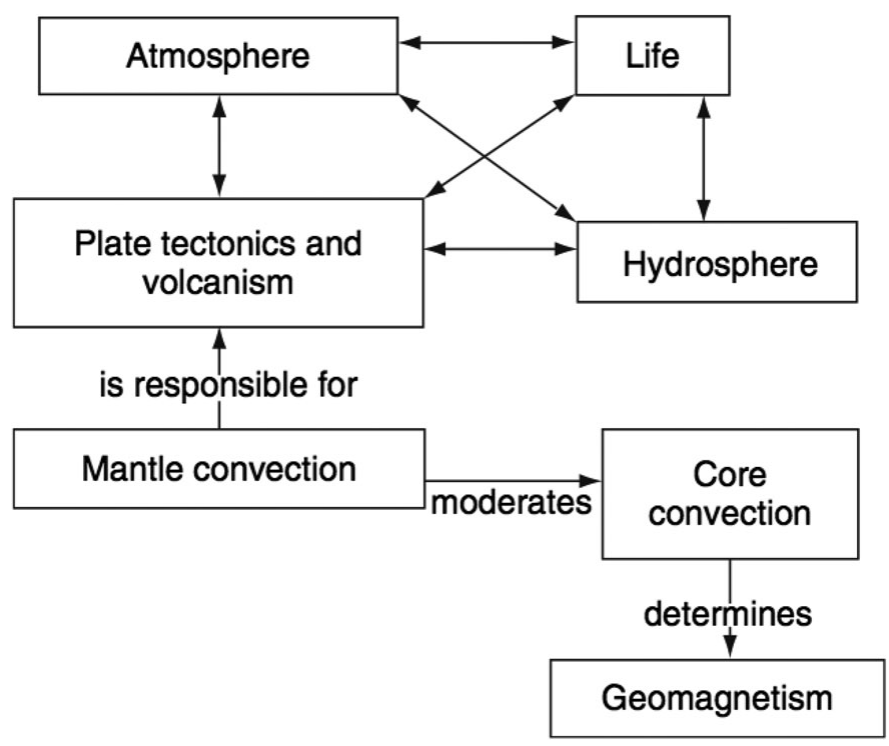

Figure 1. Interconnections between the different components of the Earth system, from the core to the atmosphere, hydrosphere and life. Over geologically long time periods, mantle convection and plate tectonics are the controlling processes. From Stevenson (2007).

\section{Mantle convection and plate tectonics}

Convection of Earth's solid rocky mantle by slow, solid-state creep is the key process that drives the evolution of Earths interior over billions of years. Mantle convection is driven by a combination of (i) radiogenic heating from ${ }^{238} \mathrm{U},{ }^{235} \mathrm{U},{ }^{232} \mathrm{Th}$ and ${ }^{40} \mathrm{~K}$ and (ii) cooling from a hot initial state arising from the accretion and differentiation processes. Mantle convection and plate tectonics are best viewed as an integrated system, with oceanic plates constituting the cold upper thermal boundary layer of the convection. The liquid outer core and the fluid atmosphere and ocean act as zero-stress, isothermal boundary conditions to this solid-state process. Geoscientists have been studying the dynamics and evolution of Earth's mantle-plate system since the discovery of plate tectonics in the late 1960s and on many topics our understanding is very good, yet many first-order questions remain [for a comprehensive account see Schubert et al. (2000) and for recent reviews of the key topics see the chapters in Bercovici (2007)].

The central role of mantle convection and plate tectonics in the long-term evolution of the entire Earth, including its core and atmosphere/hydrosphere is made clear in Fig. 1. Mantle convection controls heat loss from the metallic core, which generates the magnetic field and must be greater than some critical value for a dynamo to occur; the absence of a present-day magnetic field on Mars and Venus indicates that a dynamo-generated magnetic field is by no means guaranteed on a terrestrial planet.

Regulation of surface temperature. Long-term volatile cycling between the atmosphere/ ocean and solid interior regulates the surface environment; indeed, it is often thought that this cycling, which is facilitated by plate tectonics, is necessary for planetary habitability because negative feedback regulates the surface temperature (Walker et al. (1981)). Specifically, water, carbon dioxide and other volatiles are outgassed by volcanism, while ocean plates that reenter Earth's interior (subduct) carry with them water (incorporated into the rock) and carbonate (deposited as sedimentary rock on the oceanic floor). Most of Earth's carbon inventory (more than 99.96\%) is in the solid Earth rather than the 


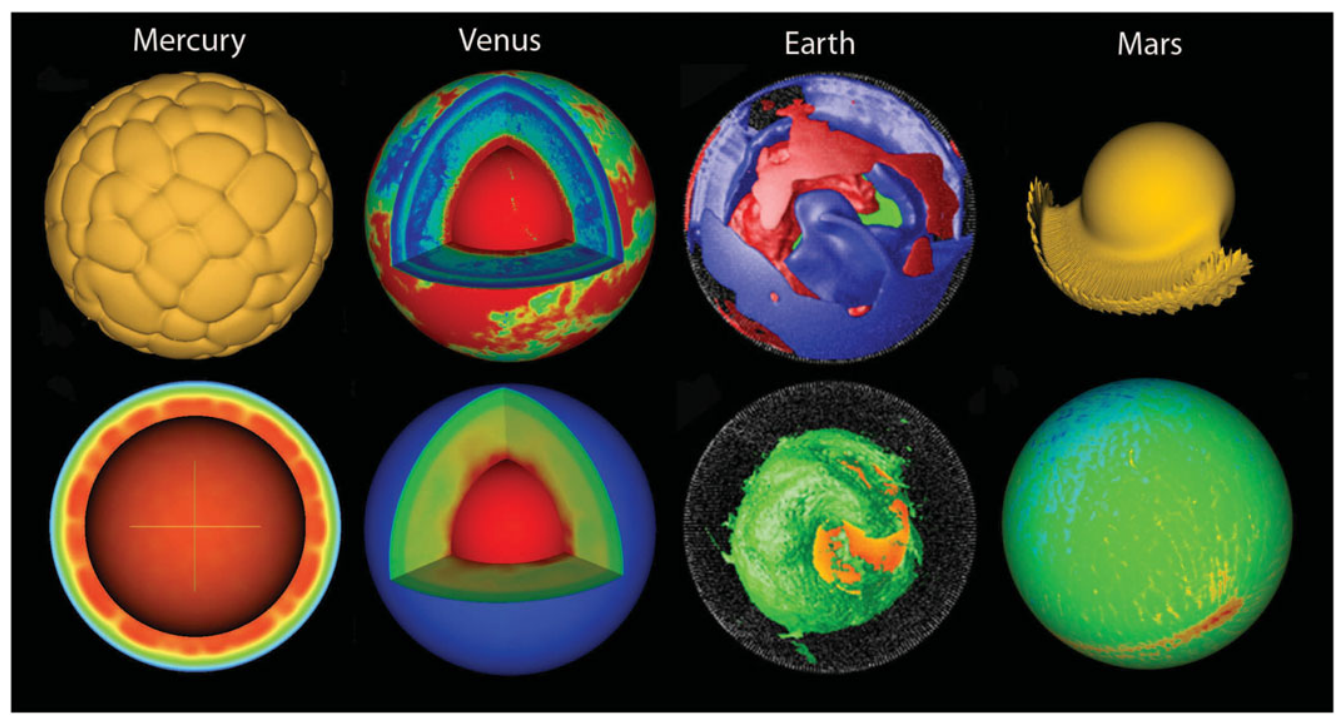

Figure 2. Numerical models of various planets in our solar system: Mercury (P.J. Tackley), Venus (a 3D version of a model in Armann \& Tackley (2012)), Earth (Nakagawa \& Tackley (2009)) and Mars (Keller \& Tackley (2009)). For Mercury temperature is plotted; cold isosurface (top) and slice (bottom). For Venus: (top) composition; crust (red) to harzburgite (blue), and (bottom) temperature. For Earth: (top) cold (blue) and hot (red) isosurfaces of residual temperature; (bottom) location of subducted MORB. For Mars: (top) hot temperature isosurface; (bottom) crustal thickness.

atmosphere-ocean system (Sleep \& Zahnle (2001)). For water it is estimated that Earths interior holds 2-6 ocean masses of water (Ahrens (1989)). Parameterized models have shown how important these cycles can be in the long-term evolution of the atmosphere. Sleep \& Zahnle (2001) found that in early Earth, carbon dioxide cycling could have resulted in low surface temperatures. In the models of Franck et al. (2002), carbon dioxide pressure falls from several bar in early Earth to the present-day value while the mass of water in the oceans calls by a factor $\sim 2.5$ since early times, and the surface temperature dropped from $350 \mathrm{~K}$ to the present-day value. The rise of atmospheric oxygen 2.5 billion years ago might be partly related to a change in oxidation state of the interior (Kump (2008), Kump et al. (2001)). While the solid Earth is thus important for buffering the surface environment, water in the mantle also has important effects on rheology that may cause interesting feedbacks (Crowley et al. (2011)).

Thermal and chemical evolution. The cooling of the planet due to cooling from a hot initial state and decay of radiogenic heat-producing elements may, as discussed above, be accompanied by atmospheric evolution, and also by internal changes. The tectonic mode may change, as discussed in the next section. Internal compositional layering may occur and evolve with time as a result of either the initial differentiation, segregation of compositionally-distinct materials that are subducted into the interior, and/or the interaction of compositional variations with solid-state phase transitions (reviewed in Tackley (2012)). Indeed, geochemical data gives unique time-integrated, though often ambiguous, constraints on interior processes, revealing the coexistence of several chemically-distinct components.

An important process, particularly in early planetary evolution, is magmatism: partial melting in the shallow mantle and subsequent upward migration of the melt to the 
surface or shallow crust can be an important heat-transport mechanism and acts as a 'thermostat' on mantle temperature, as seen in the Earth simulations of Nakagawa \& Tackley (2012), Mars simulations of Keller \& Tackley (2009) and Ogawa \& Yanagisawa (2011), Venus simulations of Armann \& Tackley (2012), and parameterized models of Kite et al. (2009).

Comparative planetology: solar system planets. Fig. 2 compares recent simulations of the evolution of Mercury, Venus, Earth and Mars performed by the lead author and coworkers, and shows how different planets can be simulated using the same basic method with different physical parameters. In the Mercury models, stagnant lid convection beneath a relatively thick stagnant lid is clear; by 4.5 billion years this is sub-critical, meaning that the velocity is decaying exponentially with time. The Venus model (a 3D version of one case reported in Armann \& Tackley (2012)) is in the episodic lid mode, in which Venus' heat is lost by bursts of plate tectonic activity interspersed by periods of stagnant lid; as a result crust is recycled and builds up above the core-mantle boundary. In the Earth models from Nakagawa \& Tackley (2009), plate tectonics occurs, again recycling crust to the CMB. In the Mars models, magmatism caused by the ridge-like plume build up a crustal dichotomy, a first-order features of Mars.

\section{Plate tectonics and scaling}

Despite the possible importance of plate tectonics on planetary habitability, as discussed above, how plate tectonics arises is still not well understood. At the present day, Earth is unique in our solar system, with the other terrestrial planets Venus and Mars instead having a stagnant lid- essentially a single plate covering the entire planet.

Temporal changes in tectonic mode. It is important to note that a planet may change its

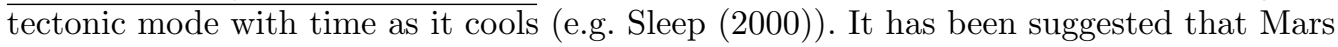
had a brief, early episode of plate tectonics (Sleep (1994)), Venus has been proposed to have episodic plate tectonics due to the near-uniform surface age (Turcotte (1993)) and some surface features have been interpreted as subduction zones (McKenzie et al. (1992), Sandwell \& Schubert (1992)). On Earth, plate tectonics as it operates today could not have operated throughout its history because a hotter mantle would make the oceanic crust thicker, making oceanic plates buoyant and unsubductable (Davies (1993)).

The plate problem. The basic problem with understanding plate tectonics is that viscosity has a strong, Arrhenius temperature-dependence, i.e. $\exp (\mathrm{E} / \mathrm{kT})$, which, using a laboratory $\mathrm{E}$ of $340 \mathrm{~kJ} / \mathrm{mol}$ and a temperature drop over the plates of $1600 \mathrm{~K}$ to $300 \mathrm{~K}$, leads to the surface viscosity being a factor $1.3 \times 10^{48}$ higher than the interior viscosity, which should make the lithosphere effectively rigid, with convection only taking place beneath it - the rigid lid or stagnant lid mode of convection.

Rock deformation. Clearly, deformation mechanisms other than viscous creep act to break the lid and generate plate boundaries. Rock deformation is complex and multi-scale (in space and time). Deformation occurs by viscous, brittle, plastic, and elastic modes, and can be nonlinear (strain-rate not proportional to stress) and history-dependent (grain size, major element composition and water content). Lengthscales range from the width of faults $(\mathrm{mm})$ to $1000 \mathrm{~s} \mathrm{~km}$, while time-scales range from seconds (earthquakes) to billions of years.

Plastic yielding. It is desirable to determine the minimum rheological complexity necessary for plate tectonics. The minimum ingredient is plastic yielding. Laboratory experiments indicate that at low pressure, the maximum differential stress that a rock sam- 


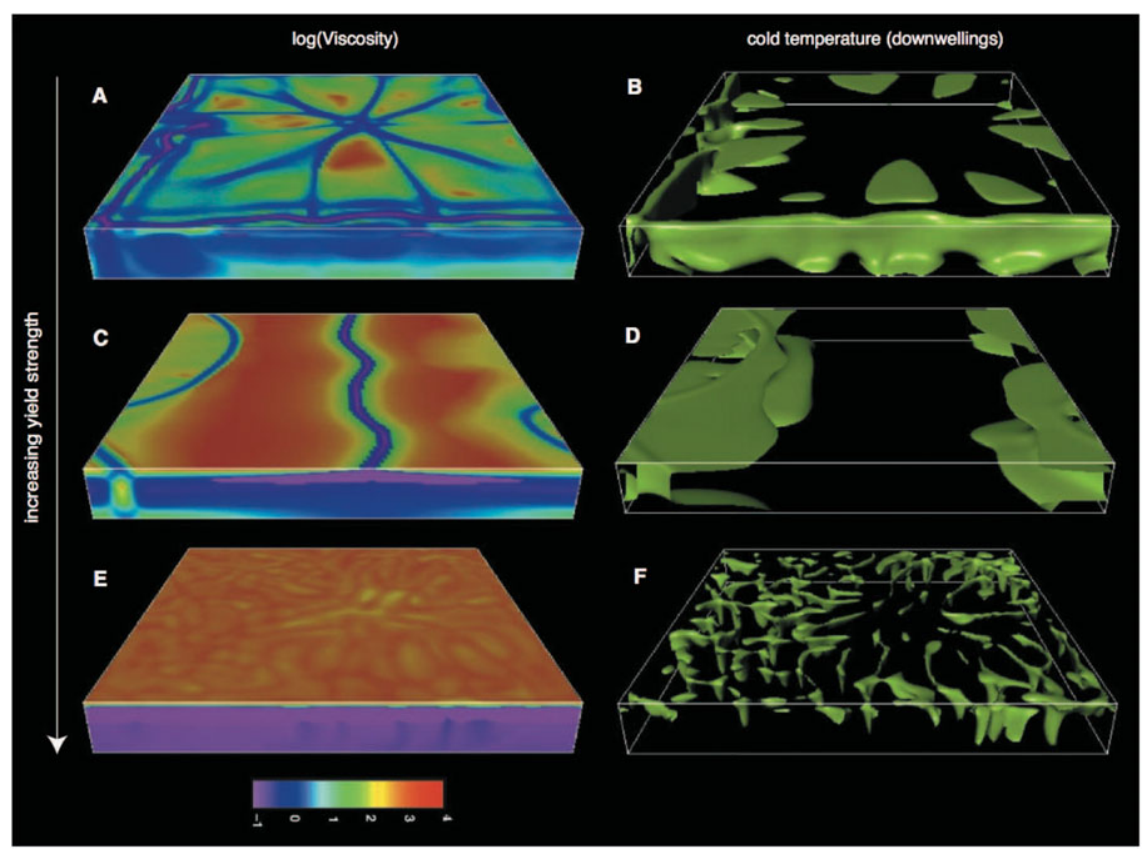

Figure 3. Plastic yielding induced plate tectonics. Parts show the influence of increasing yield stress on tectonics, from distributed deformation at low yield stress (top row) to a plate tectonics-like mode (middle row), to a stagnant lid (bottom row). Plotted are $\log 10$ of the effective viscosity (left column) and isosurface of cold temperature (right column). From Tackley (2000b).

ple can withstand increases approximately linearly with pressure, indicating frictional 'yielding', whereas at high pressure this yield stress increases only slowly with pressure, indicating ductile or semi-brittle, semi-ductile yielding (Kirby (1980)). Such experimental measurements have led to hypothesized strength profiles of the lithosphere in which the strength increases linearly in the shallow part, reaches a maximum that may occur over a certain depth range, then decreases once the high-temperature mantle is reached (Kohlstedt et al. (1995); Shimada (1993)).

Adding such plastic yielding, either as a frictional form or as a constant yield stress, to convection models with strongly temperature-dependent viscosity leads to plate tectoniclike behaviour in 2D (Moresi \& Solomatov (1998)) and in 3D (Tackley (2000a)). This is illustrated in Fig. 3. At low values of yield stress, weak plates and diffuse deformation are obtained, while intermediate yield stress values give plate tectonics-like behaviour and a high yield stress gives a stagnant lid. Between plate tectoncs and stagnant lid is an intermittent mode, with alternation between plate tectonics and a stagnant lid. A problem in such models is that the required yield stress is about 10 times lower than that measured in the laboratory: clearly the large-scale strength is much lower than the strength of a small laboratory rock sample, perhaps because small-scale features are missed, such as shear zones that may form as a result of mechanisms such as shear heating and grain-size reduction. Another problem is that subduction zones are doublesided, which can be improved using a more realistic free surface boundary condition Crameri et al. (2012).

Scaling of yielding-induced plate tectonics. Moresi \& Solomatov (1998) proposed simple analytical scaling laws to explain results from their numerical experiments of basalheated convection, and these have been verified by subsequent works. The basic finding 


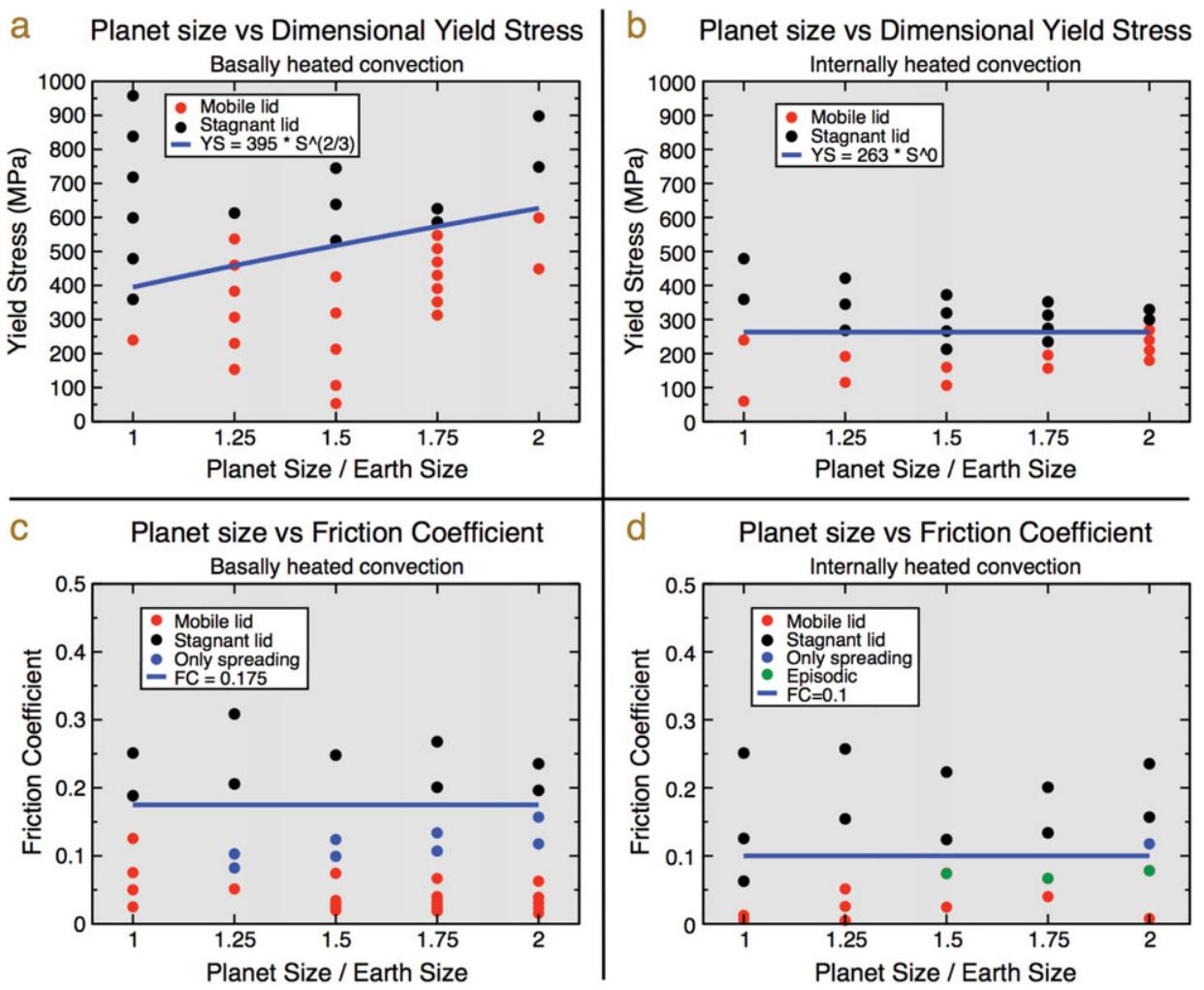

Figure 4. Scaling of critical yield stress (for obtaining plate tectonics) versus planet size for both numerical experiments (dots) and theoretical predictions (blue lines). From van Heck \& Tackley (2011). At low yield stress mobile-lid (plate tectonics) is obtained whereas at high yield stress plate tectonics is obtained. Scaling is independent of planet size. For a simple constant-viscosity planet.

is that plate tectonics is favoured at higher mantle viscosity (lower Rayleigh number), if other parameters are kept constant. This implies a transition from stagnant or episodic lid to plate tectonics as a planet cools. Other authors have looked at the influence of internal heating, finding that plate tectonics is favoured at lower internal heating rates, which again implies a transition from stagnant or episodic modes to plate tectonics as a planet evolves and radiogenic elements decay (O'Neill et al. (2007)). The presence of compositionally-distinct continents may help plate tectonics (Rolf \& Tackley (2011)] and alter various scalings (Lenardic et al. (2005).

Scaling with planet size. Several effects compete as planet size increases. Higher gravitational acceleration results in a more rapid increase of pressure with depth, implying stronger plates (O'Neill \& Lenardic (2007)) but acting in the opposite sense, a larger planet also has a higher heat flux hence thinner plates and more vigorous convection. Valencia et al. (2007) and Valencia \& O'Connell (2009) used parameterized models of a compressible planet to study the combination of the various factors on the likelihood of plate tectonics, finding that for plate tectonics is favored at larger planet size. van Heck \& Tackley (2011) studied both numerical and parameterized models of incompressible planets, studying two possible types of yield stress function (linearly increasing with pressure and constant with pressure) and two modes of heating (basal or internal) finding that for all combinations of these, plate tectonics is either equally likely or more likely on 
larger planets. If the increase of mean density with planet size is additionally taken into account, plate tectonics becomes more likely for all combinations, as in the studies of Valencia et al. (2007) and Valencia \& O'Connell (2009). These results are show in Fig. 4.

Other researchers have considered additional complexities. Korenaga (2010) included strengthening due to dehydration, predicting the same trend overall. Foley et al. (2012) assumed that the lithosphere breaks not by plastic yielding but by grainsize-damage feedback, and obtained the same trend with planet size, but also predicted that planets with a hotter surface temperature are less likely to have plate tectonics. In summary, all studies to date except for O'Neill \& Lenardic (2007) predict that plate tectonics is favoured by larger planet size, other things (particularly surface temperature and the presence of liquid water) being equal.

\section{Deep mantle dynamics}

Generally, viscosity and thermal conductivity increase with pressure while thermal expansivity decreases, resulting in lower convective vigor in the deep mantle, which, if extralopated to the largest super-Earths might, according to conventional thinking, result in no convection in their deep mantles due to the very low effective Rayleigh number (e.g. Stamenkovic et al. (2011)). In order to evaluate the influence of realistic viscosity variations on mantle dynamics in super-Earths, we have calculated the rheology using density function theory (DFT) and used it in numerical simulations of mantle convection on planets up to 10 Earth masses. These simulations further include reasonable pressure-variation of other physical properties density, thermal expansivity and thermal conductivity. Full details are reported in Tackley et al. (2013); a summary is given here. As the mantle of a super-Earth is made mostly of post-perovskite, the DFT calculations of post-perovskite activation enthalpy reported in Ammann et al. (2010) are extended to a pressure of $1 \mathrm{TPa}$, for both slowest diffusion (upper-bound rheology) and fastest diffusion (lower-bound rheology) directions. Along a $1600 \mathrm{~K}$ adiabat the upper-bound rheology would lead to a post-perovskite layer of a very high $\left(\sim 10^{30} \mathrm{~Pa} \mathrm{~s}\right)$ but relatively uniform viscosity, whereas the lower-bound rheology leads to a viscosity increase of $\sim 7$ orders of magnitude with depth; in both cases the deep mantle viscosity would be too high for convection. The calculated rheology is shown in Fig. 5. The calculated activation enthalpy
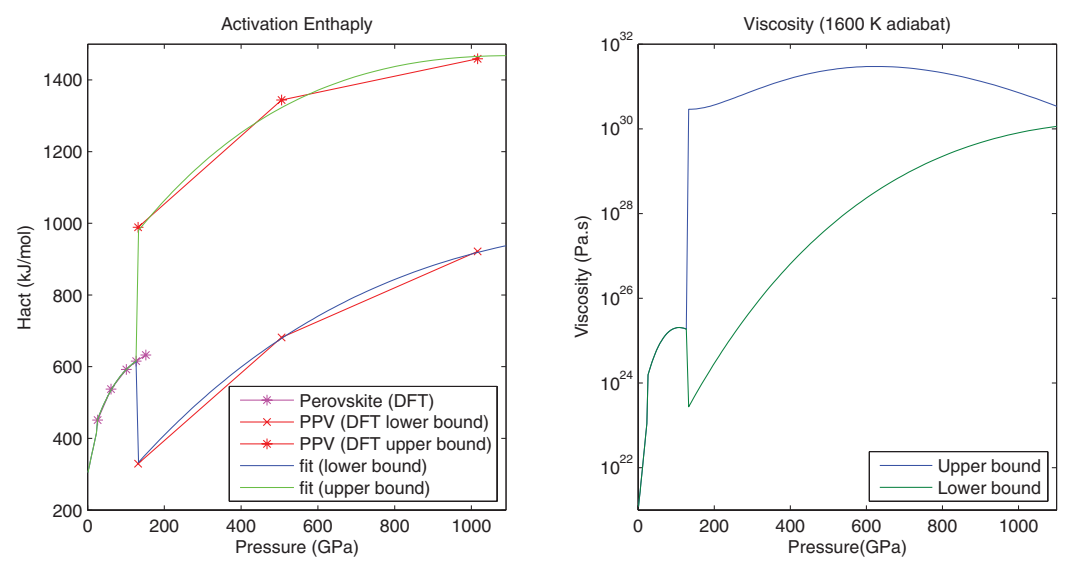

Figure 5. Pressure-dependence of activation enthapy and resulting viscosity profiles along an adiabat for lower-bound and upper-bound post-perovskite activation enthaplies. For details see Tackley et al. (2013). 


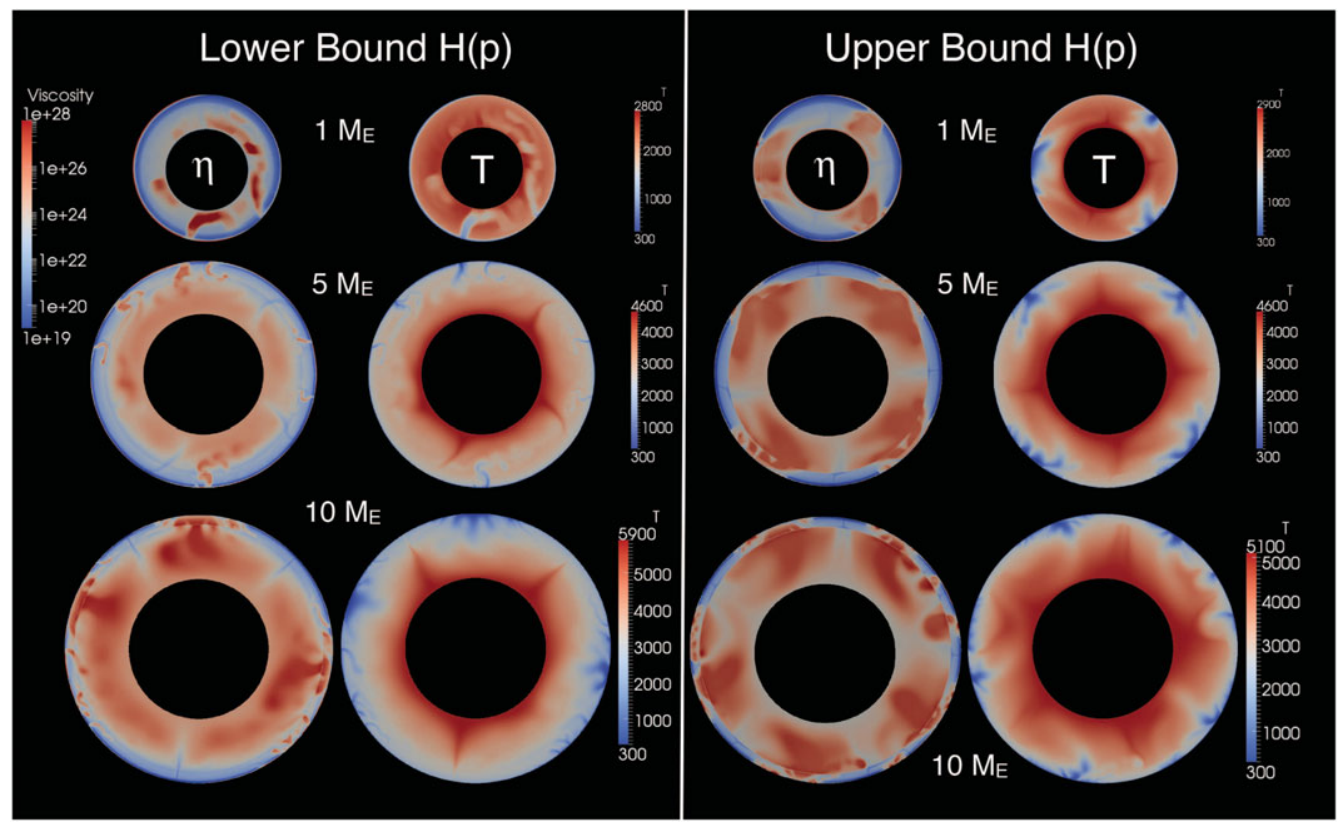

Figure 6. Plots of viscosity and temperature for $2 \mathrm{D}$ convection simulations of planets of 1,5 and 10 Earth masses, with lower-bound or upper-bound activation enthalpies. For full details see Tackley et al. (2013)

as a function of pressure is then used in numerical simulations of mantle convection and lithosphere dynamics of planets with up to ten Earth masses (Fig. 6 shows results for three sizes). The models assume a compressible mantle including depth-dependence of material properties and plastic yielding induced plate-like lithospheric behavior. This figure shows that convection in large super-Earths is characterised by large upwellings and small, time-dependent downwellings, which for large super-Earths merge into broad downwellings. It also confirms the likelihood of plate tectonics for planets with Earth-like surface conditions (temperature and liquid water).

The results show a novel self-regulation of deep mantle temperature, as can be seen in Fig. 7. The deep mantle is not adiabatic; instead feedback between internal heating, temperature and viscosity regulates the temperature such that the viscosity has the value needed to facilitate convective loss of the radiogenic heat, which results in a very hot postperovskite layer for the upper-bound rheology, a super-adiabatic post-perovskite layer for the lower-bound rheology, and an azimuthally-averaged viscosity of no more than $10^{26}$ $\mathrm{Pa}$ s. Such a self-regulation was predicted by mathematical analysis of Fowler (1993) in the parameterized (mixing-length theory) calculations of Tachinami et al. (2011) and some numerical calculations in Stamenkovic et al. (2012).

In the context of planetary evolution, if, as is likely, a super-Earth was extremely hot/molten after its formation, it is thus likely that even after billions of years its deep interior is still extremely hot and possibly substantially molten with a super basal magma ocean- a larger version of the proposal of Labrosse et al. (2007), although this depends on presently unknown melt-solid density contrast and solidus. 

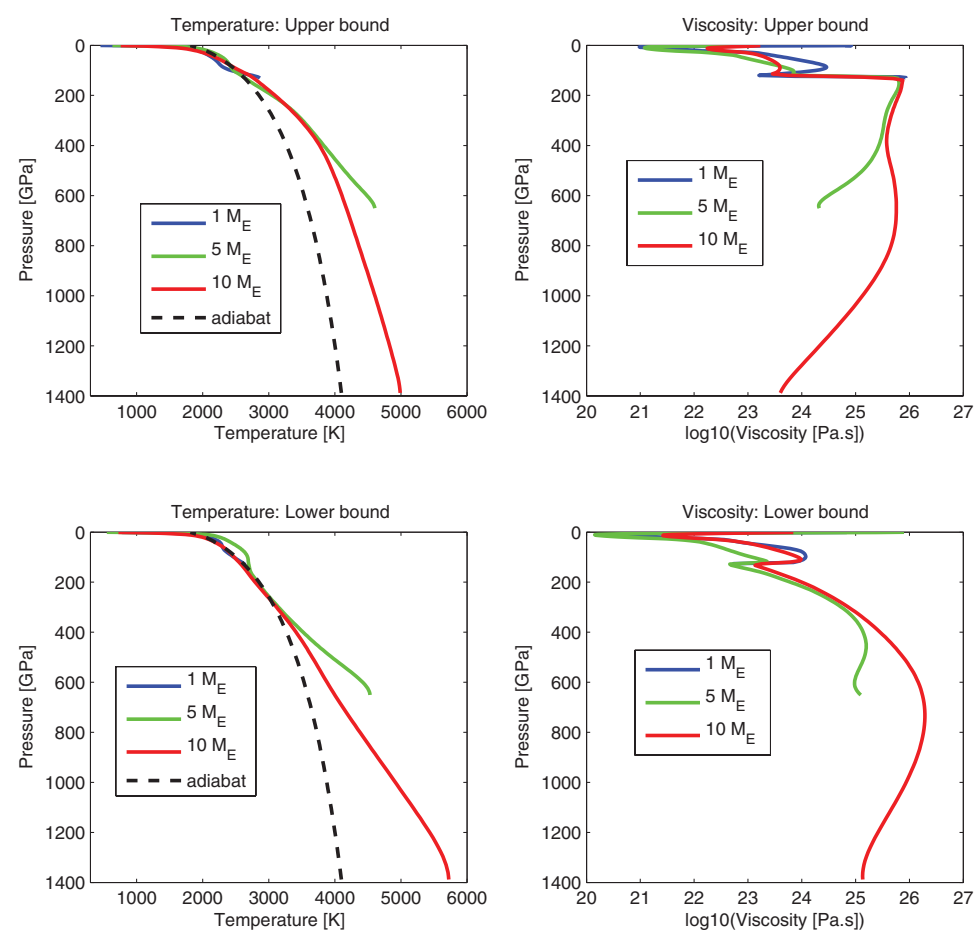

Figure 7. Profiles of temperature and viscosity.

\section{Conclusions}

Although the origin of plate tectonics on Earth is not well understood, several studies now predict that a larger terrestrial planet is equally likely or more likely to have plate tectonics, other things being equal. Two of the main factors influencing this are probably the presence or absence of liquid water in the surface environment, and surface temperature. Additionally, tectonic mode may change with time as the planet cools.

Physical properties, particularly viscosity, are expected to change at the high pressures experienced in the mantles of super Earths. Our DFT calculations of post-perovskite rheology predict a viscosity profile along an adiabat that either increases by many orders of magnitude with depth (lower bound) or increases by many orders of magnitude at the perovskite-post-perovskite transition then remains approximately constant. However, a feedback between internal heating, temperature and viscosity leads to a superadiabatic temperature profile and self-regulation of the mantle viscosity such that sluggish convection still occurs.

This paper has focussed on habitable super-Earths, i.e. with Earth-like surface temperature and water presence. If a broader range of terrestrial planets were considered, a great richness of planetary behaviour is expected, and it will be interesting to elucidate this in future studies.

\section{References}

T. J. Ahrens 1989, Nature, 342, 122 
Ammann, M. W., Brodholt, J. P., Wookey, J., \& Dobson, D. P. 2010, Nature, 465, 462

Armann, M. \& Tackley, P. J. 2012, J. Geophys. Res., 117, CiteID E12003

D. Bercovici, G. Schubert (eds.) 2007, Treatise on Geophysics Volume 7: Mantle Dynamics (Oxford and San Diego: Elsevier), pp. 505

Crameri, F., Tackley, P. J., Meilick, I., Gerya, T. V., \& Kaus, B. J. P. 2012, Geophys. Res. Lett., 39, L03306

Crowley, J. W., Grault., M., \& O'Connell, R. J. 2011, Earth Planet. Sci. Lett., 310(34), 380

Davies, G. F. 1993, Geology, 21(6), 576

Foley, B. J., Bercovici, D., \& Landuyt, W. 2012, Earth Planet. Sci. Lett., 331-332(0), 281

Fowler, A. C. 1993, Studies In Applied Mathematics, 88, 113

Franck, S., Kossacki, K. J., Von Bloh, W., \& Bounama, C. 2002, Tellus Series B-Chemical $\&$ Physical Meteorology, 54(4), 325

Keller, T. \& Tackley, P. J. 2009, Icarus, 202(2), 429

Kirby, S. H. 1980, Geophys. Res., 85, 6353

Kite, E. S., Manga, M., \& Gaidos, E. 2009, The Astrophysical Journal, 700(2), 1732

Kohlstedt, D. L., Evans, B., \& Mackwell, S. J. 1995, J. Geophys. Res., 100, 17587

Korenaga, J. 2010, Astrophys. J. Lett., 725, L43-L46

Kump, L. R. 2008, Nature, 451(7176), 277

Kump, L. R., Kasting, J. F., \& Barley, M. E. 2001, Geochem. Geophys. Geosys., 2(1), 1025

Labrosse, S., Hernlund, J. W., \& Coltice, N. 2007, Nature, 450, 866

Lenardic, A., Moresi, L. N., Jellinek, A. M., \& Manga, M. 2005, Earth Planet. Sci. Lett., 234, 317

McKenzie, D., Ford, P. G. , Johnson, C., Parsons, B., Sandwell, D., Saunders, S., \& Solomon, S. C. 1992, J. Geophys. Res., 97, 13533

Moresi, L. \& Solomatov, V. 1998, Geophys. J. Int., 133(3), 669

Nakagawa, T., Tackley, P. J., Deschamps, F., \& Connolly, J. A. D. 2009, Geophys. Geochem. Geosyst., 10, Q03004

Nakagawa, T. \& Tackley, P. J. 2010, Geophys. Geochem. Geosyst., 11, Q06001

Nakagawa, T. \& Tackley, P. J. 2012, Earth Planet. Sci. Lett., 329-330,1

O'Neill, C. \& Lenardic, A. 2007, Geophys. Res. Lett., 34, L19204

O’Neill, C., Lenardic, A., Moresi, L., Torsvik, T. H., \& Lee, C. T. A. 2007, Earth Planet. Sci. Lett., 262, 552

Ogawa, M. \& Yanagisawa, T. 2011, J. Geophys. Res., 116, E08008

Rolf, T. \& Tackley, P. J. 2011, Geophys. Res. Lett., 38, L18301

Sandwell, D. T. \& Schubert, G. 1992, Science, 257(5071), 766

Schubert, G., Turcotte, D. L., \& Olson, P. 2000, Mantle Convection in the Earth and Planets (Cambridge: Cambridge University Press)

Shimada, M., 1993, Tectonophys., 217, 55

Sleep, N. H. 1994, J. Geophys. Res., 99(E3), 5639

Sleep, N. H. 2000, J. Geophys. Res., 105(E7), 17563

Sleep, N. H. \& Zahnle, K. 2001, J. Geophys. Res., 106, 1373

Stamenkovic, V., Breuer, D., \& Spohn, T. 2011, Icarus, 216, 572

Stamenkovic, V., Noack, L., Breuer, D., \& Spohn, T. 2012, The Astrophysical Journal, 748, 41

Stevenson, D. J. 2007, Treatise on Geophysics Volume 9: Evolution of the Earth (Oxford and San Diego: Elsevier), 1

Tachinami, C., Senshu, H., \& Ida, S. 2011, The Astrophysical Journal, 726(2), 70

Tackley, P. J. 2000, Geochem., Geophys., Geosys, 1, 2000GC000036

Tackley, P. J. 2000, Science, 288, 2002

Tackley, P. J. 2011, Earth Sci. Rev., 110, 1

Tackley, P. J., Ammann, M., Brodholt, J. P. , Dobson, D. P., \& Valencia, D. 2013, Icarus, 225, 50 
Turcotte, D. L. 1993), J. Geophys. Res., 98, 17061

Valencia, D. \& O'Connell, R. J. 2009, Earth Planet. Sci. Lett., 286, 492

Valencia, D., O'Connell, R. J., \& Sasselov, D. D. 2007, Astrophys. J., 670, L45

van Heck, H. \& Tackley, P. J. 2011, Earth Planet. Sci. Lett., 310, 252

Walker, J. C. G., Hays, P. B., \& Kasting, J. F. 1981, J. Geophys. Res., 86(C10), 9776 